\title{
Capacity Definitions and Coding Strategies for General Channels with Receiver Side Information
}

\author{
Michelle Effros ${ }^{1}$ \\ Dept. of Electrical Eng., 136-93 \\ California Institute of Technology \\ Pasadena, CA 91125 USA \\ Email effros@caltech.edu
}

\author{
Andrea Goldsmith ${ }^{2}$ \\ Dept. of Electrical Eng., 136-93 \\ California Institute of Technology \\ Pasadena, CA 91125 USA \\ Email andrea@systems.caltech.edu
}

\begin{abstract}
We consider three capacity definitions for a channel with channel side information at the receiver. The capacity is the highest rate asymptotically achievable. The outage capacity is the highest rate asymptotically achievable with a given probability of decoder-recognized outage. The expected capacity is the highest expected rate asymptotically achievable using a single encoder and multiple decoders, where side information at the decoder determines which code to use. We motivate the latter definitions using the concept of maximizing reliably received rate. A coding theorem is given for each capacity.
\end{abstract}

\section{INTRODUCTION}

The rate that can be reliably transmitted in a channel with receiver side information may differ from the rate that can be reliably received. For example, consider a composite channel containing a good channel with capacity $C_{G}$ and a bad channel with capacity $C_{B}$. A coin flip determines the channel in use for all time. Let $p_{B}>0$ be the probability that the bad channel is chosen. Suppose further that only the decoder knows which channel is in use. The greatest rate that can be reliably transmitted across this channel is the channel capacity $C \leq C_{B}$ (since we must choose one coding strategy to use for both possible channel realizations). Suppose, however, that the decoder ignores the channel output when the channel is in its bad state. Then, using a code designed for the good channel, the rate reliably received is 0 when the channel is bad and $C_{G}$ when the channel is good, resulting in a total reliably received rate of $\left(1-p_{B}\right) C_{G}>C \forall p_{B}<1-C_{B} / C_{G}$. This rate is achieved using an "outage" coding strategy. Other coding strategies may achieve even higher reliably received rates.

Reliably received rate is of interest for applications where it is desirable to maximize received rate without necessarily knowing at the encoder which rate is received. Examples of such applications include systems with some acceptable outage probability, feedback channels where the receiver tells the transmitter which symbols to re-send, communications systems using multiple description source codes, or applications where lost source symbols are well predicted by surrounding samples. We here consider two strategies for maximizing received rate and consider the resulting notions of "capacity."

\section{RESUlts}

Consider a sequence of $n$-dimensional channels $\mathrm{W}=$ $\left\{W^{n}=P_{Z^{n} \mid X^{n}}\right\}_{n=1}^{\infty}$ where, for any $n>0, W^{n}$ is the conditional distribution from the input space $\mathcal{X}^{n}$ to the output

\footnotetext{
${ }^{1}$ Supported in part by NSF CAREER Award MIP-9501977, a grant from the Powell Foundation, and donations from Intel.

${ }^{2}$ Supported by ONR grant NAV-5X-N149510861 and by NSF CAREER Award NCR-9501452.
}

space $\mathcal{Z}^{n}$. Let $\mathbf{X}$ and $\mathbf{Z}$ denote the input and output processes respectively for the given sequence of channels, where each process is specified by a sequence of finite-dimensional distributions, e.g., $\mathbf{X}=\left\{X^{n}=\left(X_{1}^{(n)}, \ldots, X_{n}^{(n)}\right)\right\}_{n=1}^{\infty}$. We assume receiver side information not present at the encoder. This side information is modeled as a channel output, so $Z^{n}=\left(S^{n}, Y^{n}\right)$, where $S^{n}$ is the channel side information and $Y^{n}$ is the output of the channel described by parameter $S^{n}$. Throughout, we assume that $\mathbf{S}$ is a random variable independent of $\mathbf{X}$. We define $i\left(x^{n} ; y^{n} \mid s^{n}\right)=\log \frac{P_{X^{n}} \mid X^{n}, S^{n}\left(y^{n} \mid x^{n}, s^{n}\right)}{P_{Y^{n}} \mid S^{n}\left(y^{n} \mid s^{n}\right)}$ and $F(\alpha)=\limsup _{n \rightarrow \infty} P_{X^{n}, Y^{n}, S^{n}}\left(\frac{1}{n} i\left(X^{n} ; Y^{n} \mid S^{n}\right) \leq \alpha\right)$.

Capacity: Capacity measures the rate that can be reliably transmitted from encoder to decoder. For our general channel with receiver side information, $C=\sup _{X} \sup \{\alpha: F(\alpha)=0\}$. The above result is a simple extension of Theorems 2 and 5 of [1], or can be proved using a typicality argument.

Outage Capacity: The reliably received rate may be increased above $C$ by allowing outage. Let $P_{o}$ be the probability that a decoder, using its side information, declares an outage. Let $P_{e}$ be the probability that this decoder decodes improperly given that an outage is not declared. We say that rate $(1-q) R$ is outage- $q$ achievable if there exists a sequence of $\left(n, 2^{n R}\right)$ channel codes such that $\lim _{n \rightarrow \infty} P_{o}^{(n)} \leq q$ and $\lim _{n \rightarrow \infty} P_{e}^{(n)}=0$. The rate is $(1-q) R$ since the encoder is sending information at rate $R$ but the rate reliably received is $(1-q) R$. The outage- $q$ capacity is defined to be the supremum over all outage- $q$ achievable rates. If the side information $S^{n}$ is sufficient for determining when $i\left(X^{n} ; Y^{n} \mid S^{n}\right)$ falls below an arbitrary $\alpha \geq 0$ then the outage- $q$ capacity is bounded as $\sup _{X} \sup \{\alpha: F(\alpha)<q\} \leq \frac{C_{o: q}}{1-q} \leq \sup _{X} \sup \{\alpha: F(\alpha) \leq q\}$. Note that $\sup _{q} C_{o: q} \geq C$ for any general channel.

Expected Capacity: Another strategy for increasing reliably-received rate is to use a single encoder and a collection of decoders, parameterized by $S_{n}$, each decoding at a different rate $R_{S^{n}}$. For example, in the composite channel, the encoder uses a broadcast coding strategy for the channels in its collection and the decoder chooses which broadcast code decoder to use based on $S^{n}$ [2]. The reliably received rate is $E_{S^{n}} R_{S^{n}}$. A rate $R$ is e-achievable if there exists a sequence of channel codes such that the expected decoded rate is $R$ and the expected probability of error approaches zero. The expected capacity is the supremum of all e-achievable rates. The expected capacity $C_{e}$ satisfies

$\sup _{q} C_{o: q} \leq C_{e} \leq \sup _{X} \lim \sup _{n \rightarrow \infty} E_{X^{n} Y^{n} \mid S} \frac{1}{n} i\left(X^{n} ; Y^{n} \mid S\right)$

\section{REFERENCES}

[1] S. Verdú and T. S. Han. A general formula for channel capacity. IEEE Transactions on Information Theory, IT-40(4):11471157, July 1994.

[2] T. M. Cover. Broadcast channels. IEEE Thansactions on Information Theory, IT-18(1):2-14, January 1972. 